\title{
Wakes in stratified flow past a hot or cold two-dimensional body
}

\author{
By G. E. ROBERTSON, † J. H. SEINFELD \\ AND L. G. LEAL \\ Chemical Engineering, California Institute of Technology, Pasadena
}

(Received 3 May 1974 and in revised form 4 July 1975)

This paper considers the general problem of laminar, steady, horizontal, Oseen flow at large distances upstream and downstream of a two-dimensional body which is represented as a line source of horizontal or vertical momentum, or as a line heat source or heat dipole. The fluid is assumed to be incompressible, diffusive, viscous and stably stratified. The analysis is focused on the general properties of the horizontal velocity component, as well as on explicit calculation of the horizontal velocity profiles and disturbance stream-function fields for varying degrees of stratification. For stable stratifications, the flow fields for all four types of singularities exhibit the common feature of multiple recirculating rotors of finite thicknesses, which leads to an alternating jet structure both upstream and downstream for the horizontal velocity component and to leewaves downstream in the overall flow. The self-similar formulae for the velocity, temperature and pressure at very large distances upstream and downstream are also derived and compared with the Oseen solutions.

\section{Introduction}

The fluid-mechanical interactions of ambient temperature (or density) stratification and buoyancy-induced convection are important to a wide variety of natural and man-related phenomena in the oceans and atmosphere. The present paper is concerned with the horizontal motion of a stably stratified fluid past a heated (or cooled) two-dimensional body. The disturbance motion induced in this case is fundamentally different from that which would be observed under equivalent conditions in a homogeneous fluid. The difference is primarily a result of the additional mechanism for vorticity production in the stratified case, leading to internal waves which are responsible for standing lee-wave patterns downstream and the possibility of a greatly enhanced effect on the flow upstream, when the free-stream velocity is subcritical with respect to the horizontal phase velocity of the waves.

A large number of studies, both theoretical and experimental, have been reported for the stably stratified flow past thermally inert bodies of different shapes. Near the body where the body geometry has a direct influence on flow

$\dagger$ Present address: Chevron Oil Field Research Company, P.O. Box 446, La Habra, California 90631. 
structure, the flow has been examined in the non-diffusive case theoretically by Graebel (1969) and Janowitz (1971) and experimentally by Browand \& Winant (1972), and in the diffusive case theoretically by Freund \& Meyer (1972; see also Moore \& Saffman 1969) and experimentally by Laws \& Stevenson (1972).

Far from the body, the detailed body geometry may be considered unimportant, so that the body can be treated as a line source of horizontal and vertical momentum and heat. Janowitz $(1967,1968)$ discussed the upstream and downstream wake structure for a horizontal momentum source (i.e. the disturbance motion induced by a body which is experiencing only drag) in an unbounded, viscous, diffusive or non-diffusive stratified fluid with the inertia effects approximated using an Oseen-type linearization. A closely related analysis by List (1971) considered creeping flows induced by horizontal and vertical momentum jets in a diffusive fluid. $\dagger$ Very far upstream and downstream of the singularity, the disturbance motion becomes self-similar, with the dynamics dominated by viscous effects due to the vertical gradients of horizontal velocity and by buoyancy due to the free-stream ambient stratification. In the non-diffusive case, Long (1959) calculated a similarity solution, which describes approximately what was observed experimentally (Pao 1968; Browand \& Winant 1972) for the horizontal flow far upstream of a body which is experiencing only drag. Long (1959) could not obtain a corresponding disturbance flow downstream. However, when density diffusion was retained, Long (1962) found a new similarity solution which is valid both upstream and downstream of the body (in this respect more in accord with Laws \& Stevenson's experimental results).

In the present study, we use the methods of Janowitz (1967) to consider the two-dimensional Oseen and self-similar solutions for a line source of vertical momentum, a line source of heat, and a vertically oriented heat dipole, $t$ in the diffusive case only. In addition, for comparison purposes we also repeat portions of Janowitz' (1967) analysis for the line source of horizontal momentum. We consider both the horizontal component of the velocity (with which Janowitz and most other investigators have dealt exclusively) and the disturbance streamfunction fields which afford additional physical insight into the alternating jet structure and other interesting features of the horizontal velocity profiles. The similarities and differences in the flow structure resulting from the various types of singularities will be discussed.

\section{Physical problem and basic equations}

We consider the steady horizontal motion of an unbounded, incompressible, Newtonian fluid past an arbitrary two-dimensional body of finite cross-section. The flow is assumed to be laminar and, at large distances from the body, uniform with magnitude $U_{\infty}$. In addition, the corresponding temperature distribution far

+ Note that far from the body the disturbance motion induced by a body considered as a point momentum source is analogous to that created by a point jet.

$\$$ The motivation for our interest in the vertical heat dipole configuration will be considered in the next section. 
from the body, $T_{S}$, is assumed to increase linearly with vertical position, i.e. $T_{S}=T_{\infty}\left(1+\gamma^{\prime} y^{\prime}\right)$ with $T_{\infty}$ constant and $\gamma^{\prime}>0$, so that the ambient fluid is stably stratified. Here, $y^{\prime}$ is the dimensional vertical co-ordinate measured from the centre of the body. The corresponding downstream horizontal co-ordinate will be denoted by $x^{\prime}$. The body itself is assumed to have a uniform surface temperature, $T_{\infty}+\Delta T$, and density diffusion (i.e. thermal conduction) is explicitly included in the analysis.

The present work is specifically concerned with the velocity and temperature fields at distances from the body which are sufficiently large that the bodyinduced disturbance velocity components are small in magnitude compared with the free-stream magnitude $U_{\infty}$. In this regime, the Oseen linearization of the convective operator is applicable, and the effects of the body on the disturbance field are dominated by appropriate force and heat singularities which are concentrated at the origin, $x^{\prime}=y^{\prime}=0$. For concreteness, we consider a body which is held fixed and is thus subjected to a finite lift and/or drag force. In this case, the dominant disturbance velocity fields are induced by horizontal and vertical momentum sources. If, in addition, there is a net heat transfer to (or from) the body (i.e. $\Delta T \neq 0$ ), both a point heat source and higher-order heat singularities must also be included. In this case, the dominant contribution to the disturbance velocity and temperature fields will be due to the heat source. However, when $\Delta T=0$, so that the net heat flux from the body is zero, the dominant singularity is the vertically oriented heat dipole. Indeed if the body is symmetric about $y^{\prime}=0$, the vertically oriented heat dipole is the only relevant heat singularity. We thus concentrate here on the vertical momentum source, the heat source, and the vertically oriented heat dipole, following the earlier analysis of Janowitz $(1967,1968)$, who considered the horizontal momentum source.

The governing equations for the disturbance velocity, pressure and temperature fields are the Oseen-linearized form of the Navier-Stokes and thermal energy equations. Invoking the usual Boussinesq approximation and non-dimensionalizing these equations using the ambient fluid properties evaluated at $T=T_{\infty}$ (i.e. $y^{\prime}=0$ ), the free-stream velocity $U_{\infty}$, and the appropriate characteristic length scale (Janowitz 1967, 1968)

$$
\lambda=\left(U_{\infty} \mu_{\infty} / \beta g \rho_{\infty} T_{\infty} \gamma^{\prime}\right)^{\frac{1}{3}}
$$

we finally obtain

$$
\begin{gathered}
\frac{\partial u}{\partial x}=-\frac{\partial p}{\partial x}+R e^{-1}\left(\frac{\partial^{2} u}{\partial x^{2}}+\frac{\partial^{2} u}{\partial y^{2}}\right)-K_{1} \delta(x) \delta(y) \\
\frac{\partial v}{\partial x}=-\frac{\partial p}{\partial y}+R e^{-1}\left(\frac{\partial^{2} v}{\partial x^{2}}+\frac{\partial^{2} v}{\partial y^{2}}\right)+R e^{-1} \vartheta-K_{3} \delta(x) \delta(y) \\
\partial u / \partial x+\partial v / \partial y=0 \\
\frac{\partial \vartheta}{\partial x}+v=P e^{-1}\left(\frac{\partial^{2} \vartheta}{\partial x^{2}}+\frac{\partial^{2} \vartheta}{\partial y^{2}}\right)+K_{2 s} \delta(x) \delta(y)
\end{gathered}
$$

Here, $\beta$ is the coefficient of thermal expansion, $u$ and $v$ are the disturbance velocity components in the $x$ and $y$ (horizontal and vertical) directions, 
$\vartheta=\left(T-T_{S}\right) / \gamma^{\prime} \lambda T_{\infty}$, and $p=\left(p_{D}-p_{D \infty}\right) / \rho_{\infty} U_{\infty}^{2}$, where $p_{D}$ represents the dynamic pressure. The two dimensionless parameters which appear are the Reynolds number,

$$
R e \equiv \rho_{\infty} U_{\infty} \lambda / \mu_{\infty}=\left(\rho_{\infty}^{2} U_{\infty}^{4} / \mu_{\infty}^{2} \beta g T_{\infty} \gamma^{\prime}\right)^{\frac{1}{3}}
$$

which is a measure of the relative magnitude of inertia compared with viscous and buoyancy (due to the ambient stratification) forces, and the Péclet number,

$$
\operatorname{Pe}=\operatorname{Pr} R e \text { with } \operatorname{Pr} \equiv C_{p \infty} \mu_{\infty} / k_{\infty} .
$$

For convenience, we have incorporated the momentum and heat source contributions directly into the governing equations (cf. Janowitz 1967, 1968). The coefficients $K_{i}$ can be related to the drag, lift and net heat flux from the body, but here we shall consider only the fundamental solutions associated with $K_{i}=1$. Since the equations are linear, disturbance motions corresponding to each singularity type may be considered separately by simply decomposing the overall solutions $u, v, p$ and $\vartheta$ into the form

$$
\begin{aligned}
& u=K_{1} u_{1}+K_{2 s} R e^{-1} u_{2 s}+K_{3} u_{3}, \\
& v=K_{1} v_{1}+K_{2 s} R e^{-1} v_{2 s}+K_{3} v_{3}, \\
& p=K_{1} p_{1}+K_{2 s} R e^{-1} p_{2 s}+K_{3} p_{3}, \\
& \vartheta=K_{1} \vartheta_{1}+K_{2 s} \vartheta_{2 s}+K_{3} \vartheta_{3} .
\end{aligned}
$$

Although the vertically oriented heat dipole could have also been explicitly included in (4), a more convenient method of obtaining the corresponding disturbance fields is by simply differentiating the source solutions with respect to $y$ (Carslaw \& Jaeger 1959); e.g. $\vartheta_{2 d}=-\partial \vartheta_{2 s} / \partial y$, where the subscripts $s$ and $d$ will be used to denote heat source and dipole solutions. Finally, it may be noted that $\gamma^{\prime} \rightarrow 0$ and $(\lambda, R e, P e, \vartheta) \rightarrow \infty$ for a homogeneous fluid; hence, in this case, the governing equations must be non-dimensionalized using a different characteristic length, $\phi=\mu_{\infty} / U_{\infty} \rho_{\infty}$, and temperature scaling, $\vartheta=\left(T-T_{S}\right) / \Delta T$.

In the following sections, we describe solutions of $(1)-(4)$, subject to the freestream conditions

$$
u \rightarrow 0, \quad v \rightarrow 0, \quad p \rightarrow 0, \quad \vartheta \rightarrow 0 \quad \text { as } \quad\left(x^{2}+y^{2}\right)^{\frac{1}{2}} \rightarrow \infty .
$$

For purposes of comparison, we include not only the vertical momentum source, heat source, and heat dipole results, but also the solution for a horizontal momentum source (i.e. $K_{1}=1, K_{2}=K_{3}=0$ ) which was previously obtained by Janowitz (1967). In view of our interest in the relationships between flow structure and the degree of stratification in a 'diffusive' fluid, the explicit numerical computations which we shall describe were carried out for fixed $\operatorname{Pr}=0.7$ and various values of $R e$.

\section{Solution procedure}

The governing equations for each of the three fundamental problems which are produced by the linear decompositions $(5 a-d)$ of $(1)-(4)$ are most conveniently solved using the standard methods of Fourier transforms, as outlined in detail by 
Janowitz (1967, 1968) and List (1971). A most interesting feature of this analysis in the present case is the fact that both the solution form and a number of its general features are precisely the same for all four singularity types. Thus, denoting a typical unknown by $\zeta$ (e.g. any of $u_{1}, u_{2 s}, u_{3}, v_{1}, \ldots$ ), it may be shown that

$$
\zeta(x, y)=\pi^{-1} \int_{0}^{\infty}\left[I_{\zeta}\left(k_{2} ; x ; R e, P e\right) / 2 \pi\right] J_{\zeta}\left(k_{2} ; y\right) d k_{2}
$$

where

$$
\begin{aligned}
& I_{\zeta}\left(k_{2} ; x ; R e, P e\right)=i \int_{-\infty}^{\infty}\left[K_{\zeta}\left(k_{1}, k_{2} ; R e, P e\right) / q_{1}\left(k_{1}, k_{2} ; R e, P e\right)\right] \exp \left(i k_{1} x\right) d k_{1} \\
& \text { and } \quad q_{1}\left(k_{1}, k_{2} ; R e, P e\right)=-i\left(k_{1}^{2}+k_{2}^{2}\right)\left(i k_{1}+\frac{k_{1}^{2}+k_{2}^{2}}{R e}\right)\left(i k_{1}+\frac{k_{1}^{2}+k_{2}^{2}}{P e}\right)-i \frac{k_{1}^{2}}{R e} .
\end{aligned}
$$

The subscript $\zeta$ on $I_{\zeta}, J_{\zeta}$ and $K_{\zeta}$ is intended to indicate that the corresponding functional forms depend on the specific variable $\zeta . \dagger$ Explicit evaluation of (6)-(8) for a specific $\zeta$ is straightforward. For each $k_{2}$, the function $I_{\zeta}$ is evaluated by residue theory, after first determining the appropriate poles by solving for the roots of $q_{1}=0$ considered as a polynomial in $k_{1}$, i.e. solving for the roots of the sixth-degree polynomial

$$
\begin{aligned}
\xi^{6}+(P e+R e) \xi^{5}+\left(P e R e-3 k_{2}^{2}\right) \xi^{4}-(P e+R e) 2 k_{2}^{2} \xi^{3} \\
+\left(3 k_{2}^{4}+P e-P e R e k_{2}^{2}\right) \xi^{2}+(P e+R e) k_{2}^{4} \xi-k_{2}^{6}=0 .
\end{aligned}
$$

The physical space quantity $\zeta$ is then determined by a numerical integration over $k_{2}$.

The resulting solution clearly depends in detail upon the singularity type and on the variable in question. This is in accord with expectations. In every case, however, the $x$ dependence of the solution is completely contained in $I_{\zeta}$ and the $y$ dependence in $J_{\xi}$. Furthermore, and of greatest significance, the function $q_{1}$ and its roots are independent of $\zeta$. Janowitz (1967) has shown, for the case of a horizontal momentum source, that the general behaviour of $u_{1}$ can be deduced from a knowledge of the characteristics of the poles of $I_{\zeta}$, without actually carrying out the detailed integration of (6). Since the present analysis has shown $q_{1}$ to be independent of the singularity type, the general far-field characteristics deduced by Janowitz are also relevant for a vertical momentum source, heat source, or heat dipole at the origin. In view of the fundamental differences in the disturbance fields induced near the body, this universality of solutions in the Oseen region is both surprising and of considerable interest.

Since $q_{1}$ is a sixth-degree polynomial in $k_{1}$, its roots (and the poles of $I_{\xi}$ ) must be determined numerically for each set of values of $k_{2}, R e$ and $P e$. Janowitz (1967) has described the general features of these roots, which were also verified in the present work. Three roots, $s_{1}, s_{2}$, and $s_{3}$, have positive real parts with $0<s_{1 R}<$ $s_{2 R} \leqslant s_{3 R}$ for $k_{2}>0, \ddagger$ while the remaining three roots, $s_{4}, s_{5}$ and $s_{6}$, have negative

$\dagger$ The functions $J_{\zeta}$ and $K_{\zeta}$ are tabulated by Robertson (1975). As an example, when $\zeta=u_{1}$ we have $J_{\zeta}=k_{2}^{2} \cos k_{2} y$.

$\ddagger s_{n R}$ and $s_{n I}$ denote the real and imaginary parts of $s_{n}$. 
real parts with $0>s_{4 R} \gg s_{5 R} \geqslant s_{6 R}$ for $k_{2}>0$. For all $k_{2} \geqslant 0, s_{1}$ is real, whereas $s_{2}$ and $s_{3}$ are real for $k_{2} \geqslant k_{2}^{*}(R e, P e)$ and are complex conjugate, $s_{2}=s_{2 R}+i s_{2 I}$, $s_{3}=s_{2 R}-i s_{2 I}$, with $s_{2 I}>0$, for $0 \leqslant k_{2}<k_{2}^{*}$; the critical wavenumber $k_{2}^{*}$ increases for increasing amounts of stratification (i.e. decreasing $R e$ ). For all $k_{2} \geqslant 0, s_{4}$ is real, whereas $s_{5}$ and $s_{6}$ may be real or complex conjugate, depending on the values of $R e$ and $P e$. Finally, $s_{1}$ and $s_{2 R}$ increase with increasing $k_{2} \geqslant 0$, while $s_{2 I}$ decreases; $s_{2 R}$ and $s_{2 I}$ increase for decreasing $R e$ and $s_{1} \sim k_{2}^{3} / P e^{\frac{1}{2}}$ for $k_{2}$ near zero. In contrast, the real parts of all three roots $s_{4}, s_{5}$ and $s_{6}$ decrease for increasing $k_{2} \geqslant 0$, with $s_{4} \sim-k_{2}^{3} / P e^{\frac{1}{2}}$ for $k_{2}$ near 0 . The function $I_{\zeta}$ for $x>0$ (downstream of the body) is evaluated using the poles with $s_{n R}>0$, while the region $x<0$ requires the poles with $s_{n R}<0$.

In the following discussion, we shall focus attention on the horizontal velocity components $u_{1}, u_{2 s}, u_{2 d}$ and $u_{3}$. Taking account of the nature of the poles $s_{1}, s_{2}, \ldots, s_{6}$, the general expression (7) for $I_{\zeta}$, with $\zeta=u_{1}, u_{2 s}, u_{2 d}$, or $u_{3}$, may be written in the more specific forms

$$
\begin{aligned}
(2 \pi)^{-1} I_{\zeta}=A_{\zeta} & R e \exp \left(-s_{1} x\right) /\left\{\left|s_{2}-s_{1}\right|^{2}\left(s_{1}-s_{4}\right)\left(s_{1}-s_{5}\right)\left(s_{1}-s_{6}\right)\right\} \\
+ & R e \exp \left(-s_{2 R} x\right) \sum_{n=0}^{3} B_{n \zeta} \sin \left(s_{2 I} x+\theta_{1}+\theta_{4}+\theta_{5}+\theta_{6}-n \theta_{2}\right) \\
& \times\left\{s_{2 I}\left|s_{2}-s_{1}\right|\left|s_{2}-s_{4}\right|\left|s_{2}-s_{5}\right|\left|s_{2}-s_{6}\right|\right\}^{-1}
\end{aligned}
$$

for $x>0$ and $0 \leqslant k_{2}<k_{2}^{*}$;

$$
\begin{aligned}
(2 \pi)^{-1} I_{\zeta}=C_{\zeta} & R e \exp \left(-s_{1} x\right) /\left\{\left(s_{1}-s_{2}\right)\left(s_{1}-s_{3}\right)\left(s_{1}-s_{4}\right)\left(s_{1}-s_{5}\right)\left(s_{1}-s_{6}\right)\right\} \\
& +D_{\zeta} R e \exp \left(-s_{2} x\right) /\left\{\left(s_{2}-s_{1}\right)\left(s_{2}-s_{3}\right)\left(s_{2}-s_{4}\right)\left(s_{2}-s_{5}\right)\left(s_{2} s-{ }_{6}\right)\right\} \\
& +E_{\zeta} \operatorname{Re} \exp \left(-s_{3} x\right) /\left\{\left(s_{3}-s_{1}\right)\left(s_{3}-s_{2}\right)\left(s_{3}-s_{4}\right)\left(s_{3}-s_{5}\right)\left(s_{3}-s_{6}\right)\right\}
\end{aligned}
$$

for $x>0$ and $k_{2} \geqslant k_{2}^{*}$;

$$
\begin{aligned}
(2 \pi)^{-1} I_{\zeta}=F_{\zeta} & R e \exp \left(-s_{4} x\right) /\left\{\left(s_{4}-s_{1}\right)\left|s_{2}-s_{4}\right|^{2}\left(s_{4}-s_{5}\right)\left(s_{4}-s_{6}\right)\right\} \\
& +G_{\zeta} R e \exp \left(-s_{5} x\right) /\left\{\left(s_{5}-s_{1}\right)\left|s_{2}-s_{5}\right|^{2}\left(s_{5}-s_{4}\right)\left(s_{5}-s_{6}\right)\right\} \\
& +L_{\zeta} R e \exp \left(-s_{6} x\right) /\left\{\left(s_{6}-s_{1}\right)\left|s_{2}-s_{6}\right|^{2}\left(s_{6}-s_{4}\right)\left(s_{6}-s_{5}\right)\right\}
\end{aligned}
$$

for $x<0$ and $0<k_{2}<k_{2}^{*}$;

$$
\begin{aligned}
(2 \pi)^{-1} I_{\zeta}=M_{\zeta} & R e \exp \left(-s_{4} x\right) /\left\{\left(s_{4}-s_{1}\right)\left(s_{4}-s_{2}\right)\left(s_{4}-s_{3}\right)\left(s_{4}-s_{5}\right)\left(s_{4}-s_{6}\right)\right\} \\
& +N_{\zeta} \operatorname{Re} \exp \left(-s_{5} x\right) /\left\{\left(s_{5}-s_{1}\right)\left(s_{5}-s_{2}\right)\left(s_{5}-s_{3}\right)\left(s_{5}-s_{4}\right)\left(s_{5}-s_{6}\right)\right\} \\
& +P_{\zeta} \operatorname{Re} \exp \left(-s_{6} x\right) /\left\{\left(s_{6}-s_{1}\right)\left(s_{6}-s_{2}\right)\left(s_{6}-s_{3}\right)\left(s_{6}-s_{4}\right)\left(s_{6}-s_{5}\right)\right\}
\end{aligned}
$$

for $x<0$ and $k_{2} \geqslant k_{2}^{*}$;

where

$$
\begin{gathered}
\left|s_{2}-s_{i}\right|=\left[\left(s_{2 R}-s_{i}\right)^{2}+s_{2 I}^{2}\right]^{\frac{1}{2}}, \quad i=1,4,5,6 ; \\
\left|s_{2}\right|=\left(s_{2 R}^{2}+s_{2 I}^{2}\right)^{\frac{1}{2}} ; \\
\theta_{2}=\tan ^{-1}\left(s_{2 I} l s_{2 R}\right) ; \\
\theta_{i}=\tan ^{-1}\left[s_{2 I} /\left(s_{2 R}-s_{i}\right)\right], \quad i=1,4,5,6 .
\end{gathered}
$$

The coefficients $A_{\zeta}, B_{0 \zeta}, B_{1 \zeta}, B_{2 \zeta}, B_{3 \zeta}, C_{\zeta}, D_{\zeta}, E_{\zeta}, P_{\zeta}, G_{\zeta}, L_{\zeta}, M_{\zeta}, N_{\zeta}$ and $P_{\zeta}$, which are functions of $\zeta, k_{2}, s_{1}, s_{2}, s_{3}, s_{4}, s_{5}, s_{6}$ and $P e$, but not of $x$, are tabulated by 


$$
\begin{aligned}
& u_{1}=-R P e^{-\frac{1}{6}}|x|^{-\frac{2}{3}} C_{1} \\
& u_{2 d}=\mp R P e^{\frac{1}{3}}|x|^{-1} C_{2} \\
& u_{2 s}= \pm R P e^{\frac{1}{3}}|x|^{-\frac{2}{3}} S_{1} \\
& u_{3}=\mp R P e^{-\frac{1}{3}}|x|^{-\frac{4}{3}} S_{3} \\
& p_{1}=\mp(2 \pi)^{-1} P e^{\frac{1}{6}}|x|^{-\frac{1}{3}} C_{0} \\
& p_{2 d}=-(2 \pi)^{-1} P e^{\frac{5}{6}}|x|^{-\frac{3}{3}} C_{1} \\
& p_{2 s}=+(2 \pi)^{-1} P e^{\frac{2}{3}}|x|^{-\frac{1}{3}} S_{0} \\
& p_{3}=-(2 \pi)^{-1}|x|^{-1} S_{2}
\end{aligned}
$$

$$
\begin{aligned}
& v_{1}=\mp R P e^{-\frac{1}{3}}|x|^{-\frac{4}{3}} S_{3} \\
& v_{2 d}=-R P e^{\frac{1}{3}}|x|^{-\frac{5}{3}} S_{4} \\
& v_{2 s}=-R P e^{\frac{1}{6}}|x|^{-\frac{4}{3}} C_{3} \\
& v_{3}=+R P e^{-\frac{1}{2}}|x|^{-2} C_{5} \\
& \vartheta_{1}= \pm R P e^{\frac{1}{3}}|x|^{-\frac{2}{3}} S_{1} \\
& \vartheta_{2 d}=+(2 \pi)^{-1} P e|x|^{-1} S_{2} \\
& \vartheta_{2 s}=+(2 \pi)^{-1} P e^{\frac{5}{6}}|x|^{-\frac{2}{3}} C_{1} \\
& \vartheta_{3}=-R P e^{\frac{1}{6}}|x|^{-\frac{4}{3}} C_{3}
\end{aligned}
$$

TABLE 1. Self-similar diffusive wake solutions.

(For notation see (14) et seq.)

Robertson (1975). For additional physical insight, we also calculate the disturbance stream-function fields $\psi_{i}\left(i=1,2_{s}, 2_{d}, 3\right)$, where

$$
\begin{gathered}
\tilde{\psi}=y+K_{1} \psi_{1}+K_{2 s} R e^{-1} \psi_{2 s}+K_{3} \psi_{3}, \\
\tilde{u}=\partial \tilde{\psi} / \partial y, \quad \tilde{v}=-\partial \psi_{r} / \partial x, \\
u_{i}=\partial \psi_{i} / \partial y, \quad v_{i}=-\partial \psi_{i} / \partial x, \quad i=1,2_{s}, 2_{d}, 3 .
\end{gathered}
$$

\section{Solutions}

\section{Self-similar solutions}

Very far downstream and upstream of each singularity type, the integrand for $\zeta$ is non-negligible only near $k_{2}=0$; for example, expressions (10) and (11) for $I_{u_{i}}, i=1,2_{s}, 2_{d}, 3$, are dominated by the first terms of $(10 a)$ and (11 $\left.a\right)$ respectively, evaluated near $k_{2}=0$. For $k_{2} \equiv 0, s_{1} \equiv s_{4} \equiv 0$ and (9) yields $\left|s_{2}\right|^{2} s_{5} s_{6}=P e$. Since $A_{u_{1}} \approx-k_{2}^{2}$ near $k_{2}=0$ and $J_{u_{1}}=k_{2}^{2} \cos k_{2} y$ (Robertson 1975), the expression for $u_{1}$ very far downstream becomes

$$
u_{1}(x, y)=R P e^{-\frac{1}{2}} \int_{0}^{\infty} k_{2} \cos k_{2} y \exp \left(-k_{2}^{3} x / P e^{\frac{1}{2}}\right) d k_{2},
$$

where $R=(2 \pi)^{-1} R e$. As Janowitz has noted, this solution is identical to Long's (1962) similarity solution for the far-wake motion generated by a source of horizontal momentum in a diffusive stratified fluid. Indeed this is simply shown by substituting $s^{3}$ for $k_{2}^{3} x / P e^{\frac{1}{2}}$ in the above exponential to yield

$$
u_{1}=-R P e^{-\frac{1}{y}}|x|^{-\frac{2}{3}} C_{1}(\eta), \text { where } \eta=P e^{\frac{1}{8}} y|x|^{-\frac{1}{3}},
$$

and $C_{1}(\eta)$ is defined by the following equation. One can analogously obtain selfsimilar solutions for all of the other physical variables and singularity types, $\zeta$, and these are listed in table 1 , in which

$$
C_{n}=C_{n}(\eta)=\int_{0}^{\infty} s^{n} \cos \eta s \exp \left(-s^{3}\right) d s
$$

and $S_{n}(\eta)$ is the same with sin in place of cos. The presence of two signs in some of the expressions corresponds to $x>0$ (upper sign) and $x<0$ (lower sign). The self-similar solutions for $u_{1}, v_{1}, p_{1}$, and $\vartheta_{1}$ were previously calculated by 

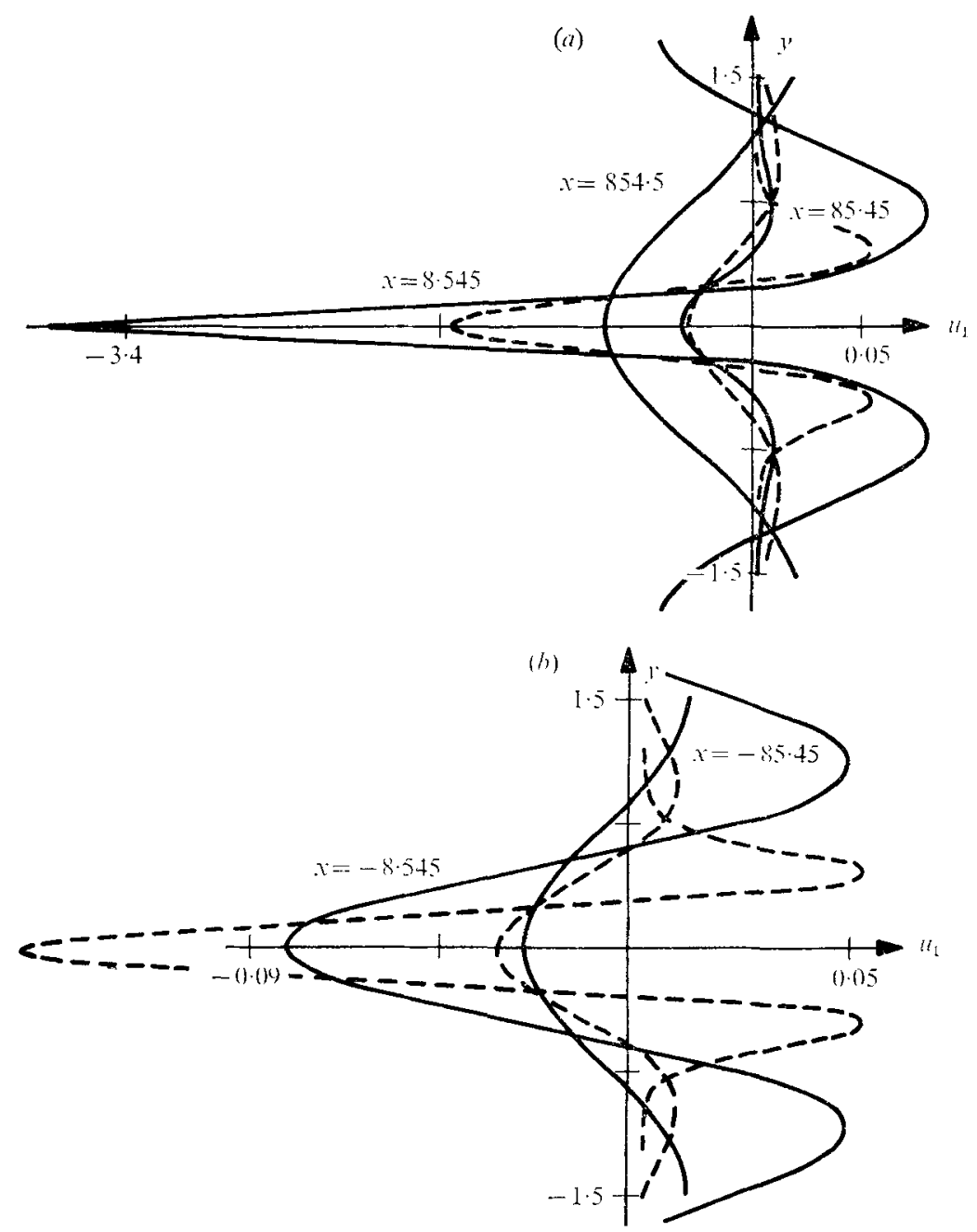

Figure 1. $u_{1}$ as a function of $y$ for several fixed values of $x$ and $\operatorname{Re}=11 \cdot 68, \operatorname{Pr}=0 \cdot 7$. - Oseen solutions; - - - self-similar solutions. (a) $x>0$. (b) $x<0$.

Janowitz (1967) and Long (1962), and have been included here for comparison purposes. Plots of the self-similar solutions for $u_{1}, u_{2 s}, u_{2 d}$ and $u_{3}$ are presented here in figures 1-8; a complete set of plots of the similarity functions may be found in Robertson (1975).

It is significant that for each singularity type the self-similar regime is dominated by the same physical processes. Indeed, one can easily show that the self-similar profiles, which were obtained as approximations of the Oseen solutions at very large distances, are also exact solutions of the equations

$$
\begin{gathered}
\partial p / \partial x=R e^{-1} \partial^{2} u / \partial y^{2}-K_{1} \delta(x) \delta(y) \\
\partial p / \partial y=R e^{-1} \vartheta-K_{3} \delta(x) \delta(y) \\
\partial u / \partial x+\partial v / \partial y=0 \\
v=P e^{-1} \partial^{2} \vartheta / \partial y^{2}+K_{2 s} \delta(x) \delta(y)
\end{gathered}
$$



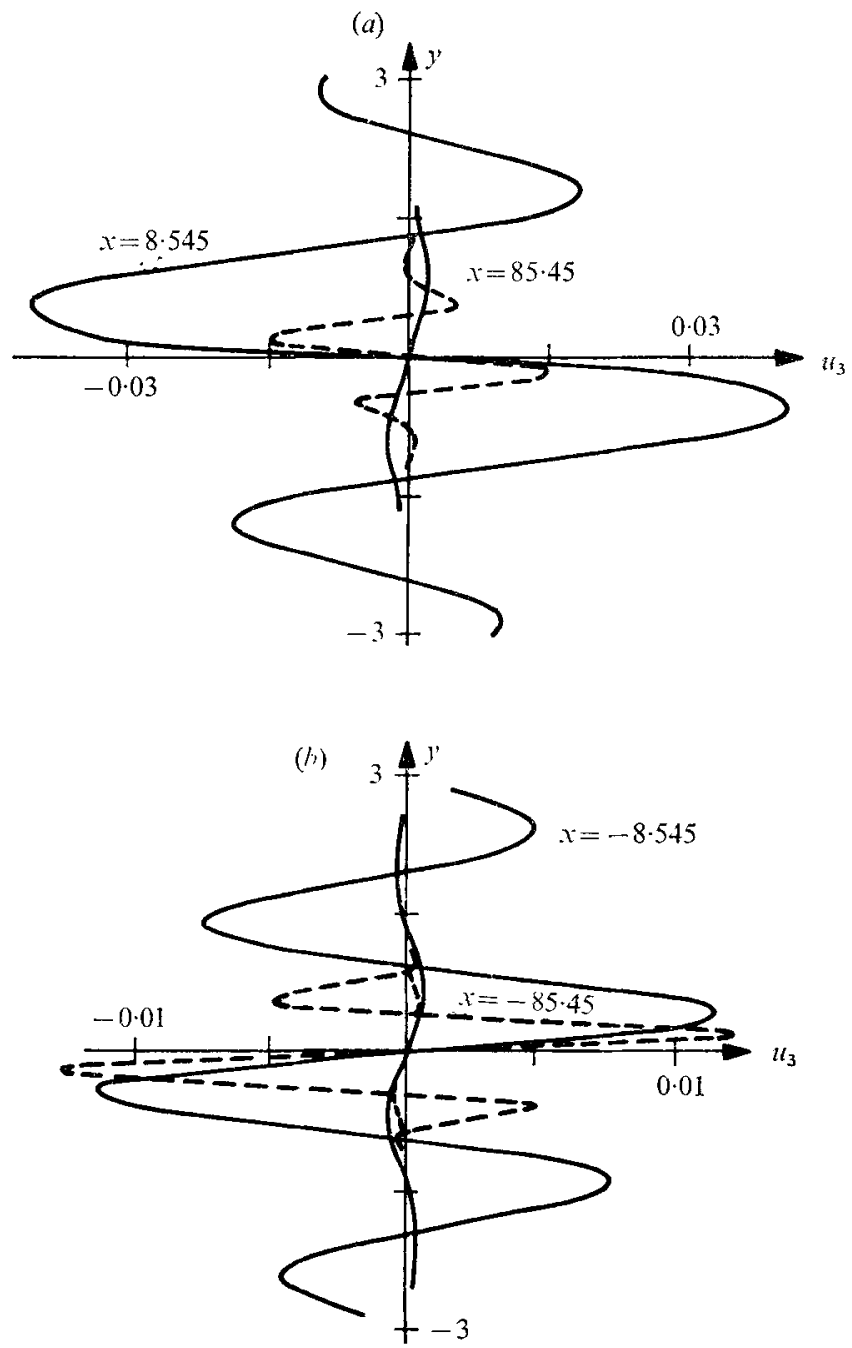

FIGURE 2. $u_{3}$ as a function of $y$ for several fixed values of $x$ and $R e=11 \cdot 68, \operatorname{Pr}=0.7$. $\ldots$, Oseen solutions; - - - , self-similar solutions. (a) $x>0$. (b) $x<0$.

which were solved originally by Long (1962) for $K_{2 s}=K_{3}=0, K_{1} \neq 0$. Note that, in all cases, inertia effects are unimportant in this far-wake self-similar region, although significant in the Oseen regime which lies closer to the singularity (i.e. the body). +

In spite of the fact that the basic physics relating $u, v, p$ and $\vartheta$ is similar in all cases, the detailed differences in the flows induced nearer the body by the various types of singularities are still rather strongly reflected in the solutions as far away as the self-similar regime. This is true not only in the detailed profiles, but particularly in the dependence on the parameters $R e$ and $P e$, and in the rates of decrease of the magnitudes of the velocity, pressure and temperature profiles

$\dagger$ Criteria, in terms of the required distances from the body, for the validity of the Oseen and self-similar solutions are presented in the appendix. 


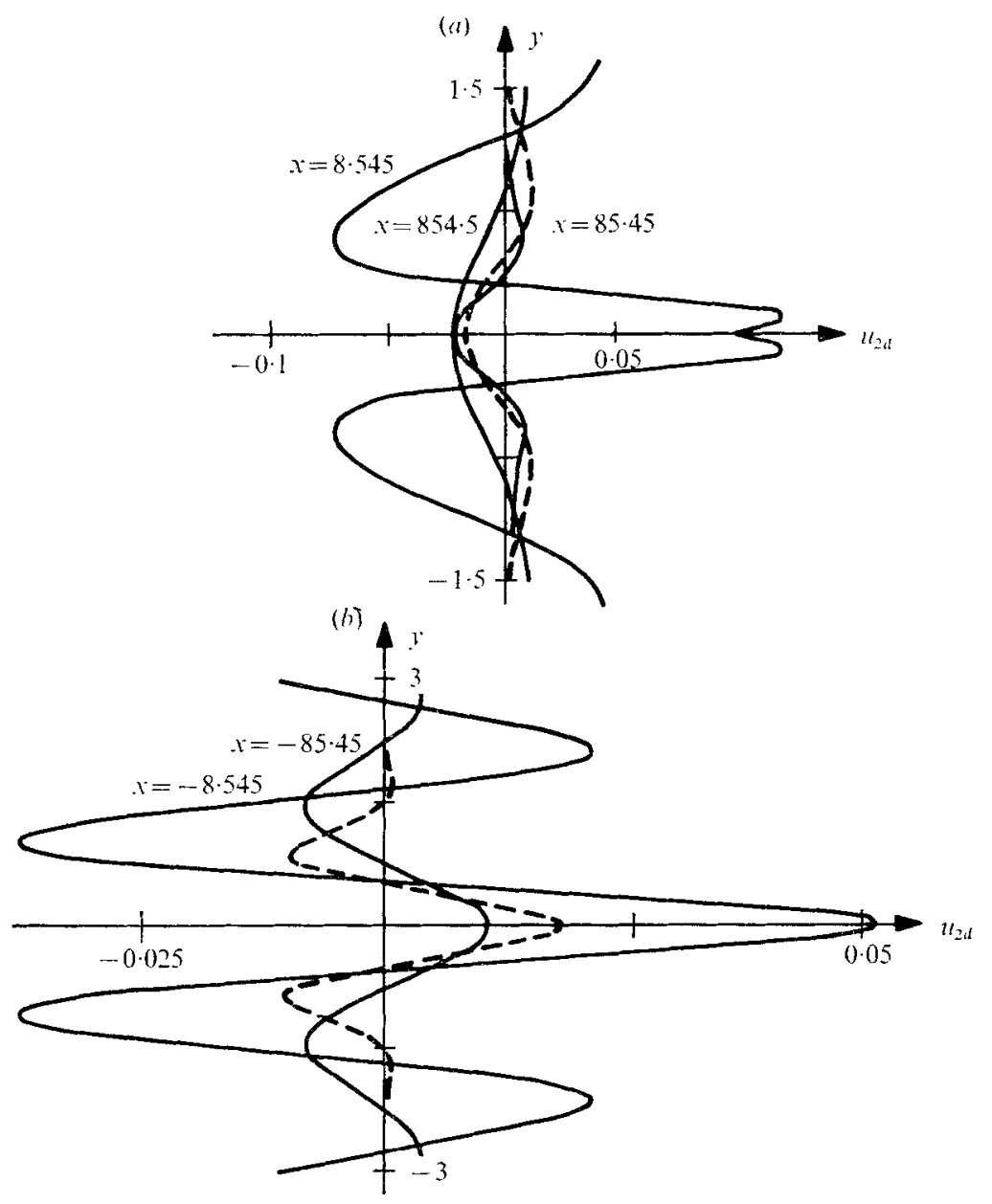

Figure 3. $u_{2 d}$ as a function of $y$ for several fixed values of $x$ and $R e=11 \cdot 68, \operatorname{Pr}=0 \cdot 7$. $\ldots$ Oseen solutions; - - - , self-similar solutions. $(a) x>0$. (b) $x<0$.

with increasing distances $|x|$. In the case of the horizontal momentum source, the main peaks in the similarity profiles upstream and downstream result directly from the horizontal flow induced at the origin (for $K_{1}>0$, this flow is from right to left). Not surprisingly, the horizontal velocity component $u_{1}$ is dominant over the vertical component $v_{1}$. In the cases of the vertical momentum source and the heat source, the flow induced at the body is dominantly vertical, upward for $K_{2 s}>0$ and downward for $K_{3}>0$. However, as we shall see more clearly in the next section, the vertical near-body motions are constrained by the ambient density stratification, and converted efficiently into strong horizontal motions which are antisymmetric about the $x$ axis $(y=0)$. As in the previous case, the magnitude of the horizontal velocity component is larger than that of the vertical component by $O\left(|x|^{+\frac{2}{3}}\right)$ in this distant self-similar regime. The antisymmetry and sign of the induced horizontal motions in the far wake are easily understood. For example, considering $u_{3}$, the ambient stratification causes the 

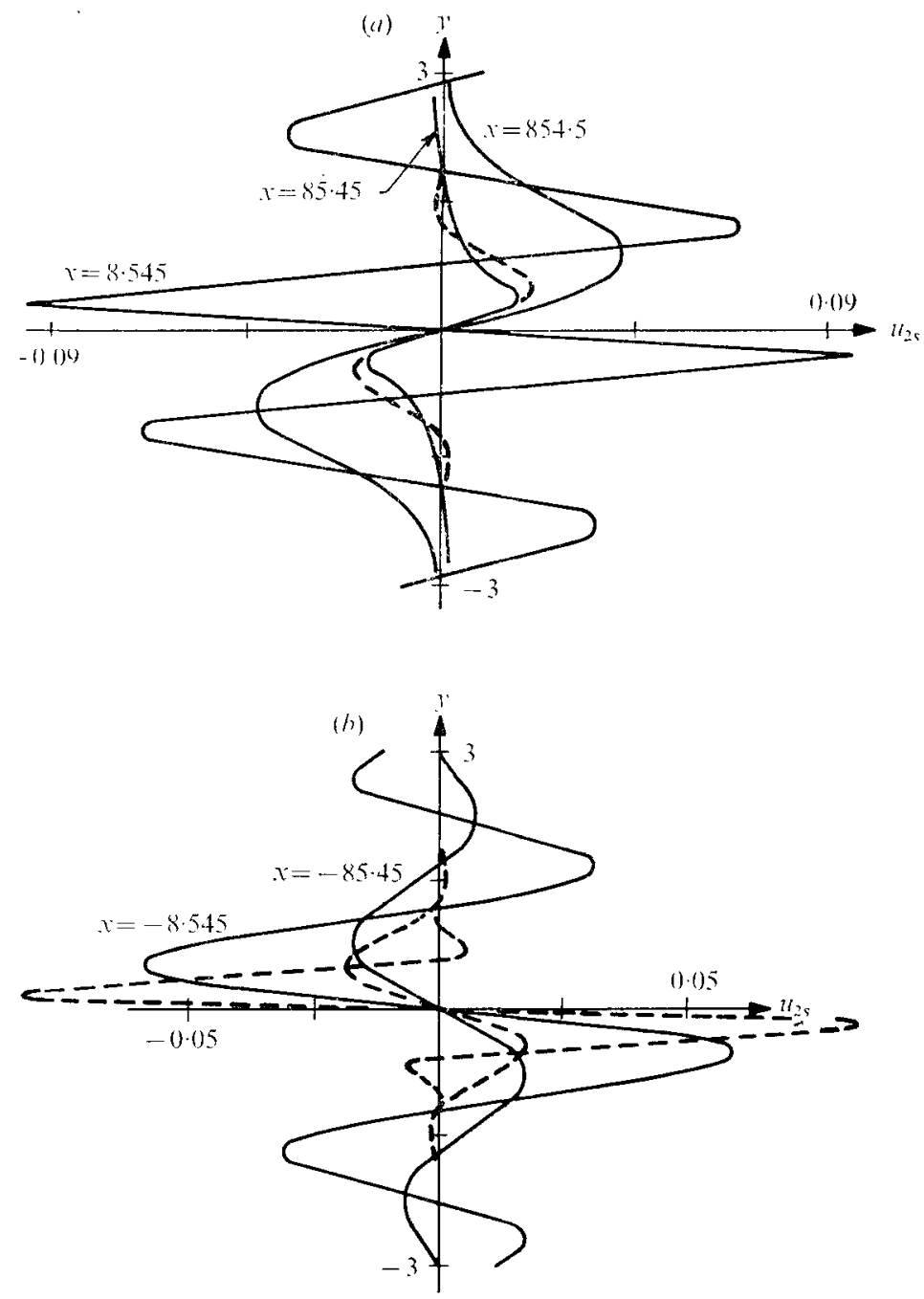

Figure 4. $u_{2 s}$ as a function of $y$ for several fixed values of $x$ and $R e=11 \cdot 68, \operatorname{Pr}=0 \cdot 7$. - , Oseen solutions; - - - , self-similar solutions, $(a) x>0$. (b) $x<0$.

downward vertical motions near the body to be turned into both upstream and downstream jet-like motions. Below the plane $y=0$ these motions will be outward, whereas above they must be inward in order to satisfy the entrainment requirements for the near-body flow. One surprising feature, from the physical point of view, is the precise upstream-downstream symmetry in the self-similar regime. Mathematically, however, it is obvious from (15), in which inertia effects are neglected, that the solutions must exhibit this feature. Nearer the body, however, where convective inertia effects cannot be neglected for finite $R e$, the velocity and temperature fields would be expected to show some degree of upstream-downstream asymmetry, and this will become apparent in the full Oseen solutions which we shall discuss next. Finally, the horizontal velocity profile for a heat dipole appears qualitatively similar in shape to the profile for a 


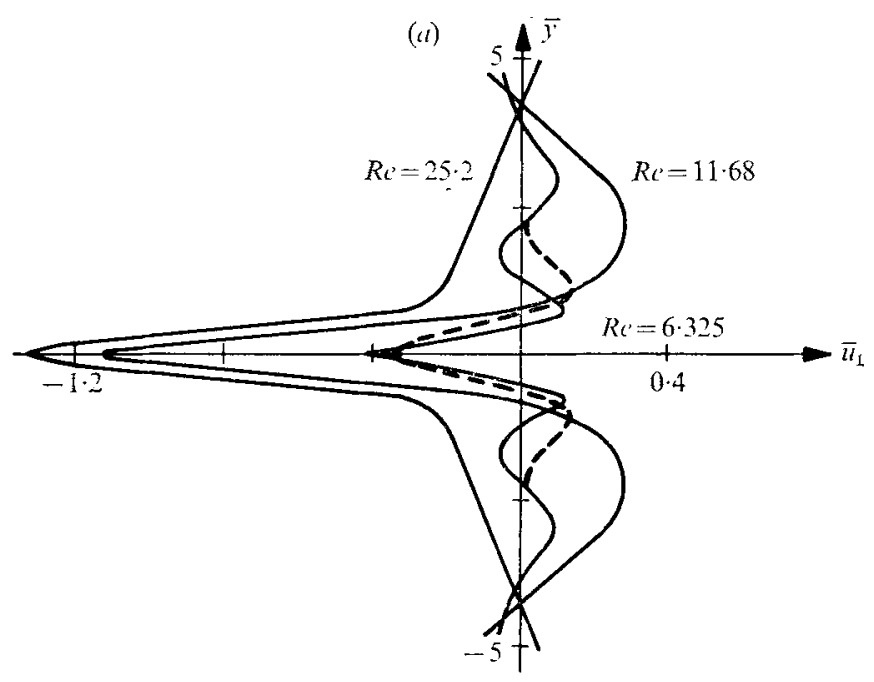

(b)

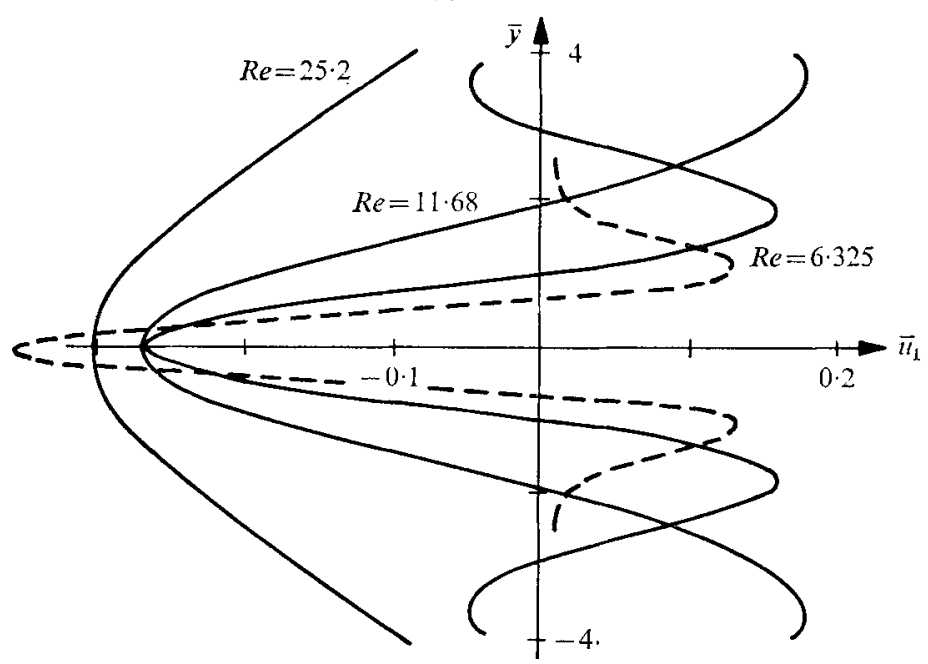

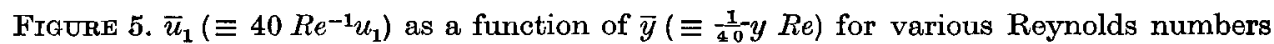
and $\operatorname{Pr}=0 \cdot 7$. - Oseen solutions for $R e=6 \cdot 325,11 \cdot 68,25 \cdot 2 ;-\cdots--$, self-similar solution for $R e=6 \cdot 325$.
(a) $\bar{x}=\frac{1}{40} x R e=2 \cdot 5$.
(b) $\bar{x}=\frac{1}{40} x R e=-2 \cdot 5$

horizontal momentum source. For a sufficiently simple body geometry, such as the horizontal flat plate (cf. Robertson, Seinfeld \& Leal 1973), this similarity appears quite natural since the local density gradients induced by the body acting as a heat dipole produce an effective horizontal pressure gradient at the body surface which acts to accelerate the fluid in the horizontal direction. The buoyancy effects which act to produce vertical motions locally produce no net vertical motion for the heat dipole. Thus, for a simple body geometry, the heat dipole appears as an effective source of horizontal momentum. Generalization of these simple ideas to more complicated body geometries is, however, not obvious 

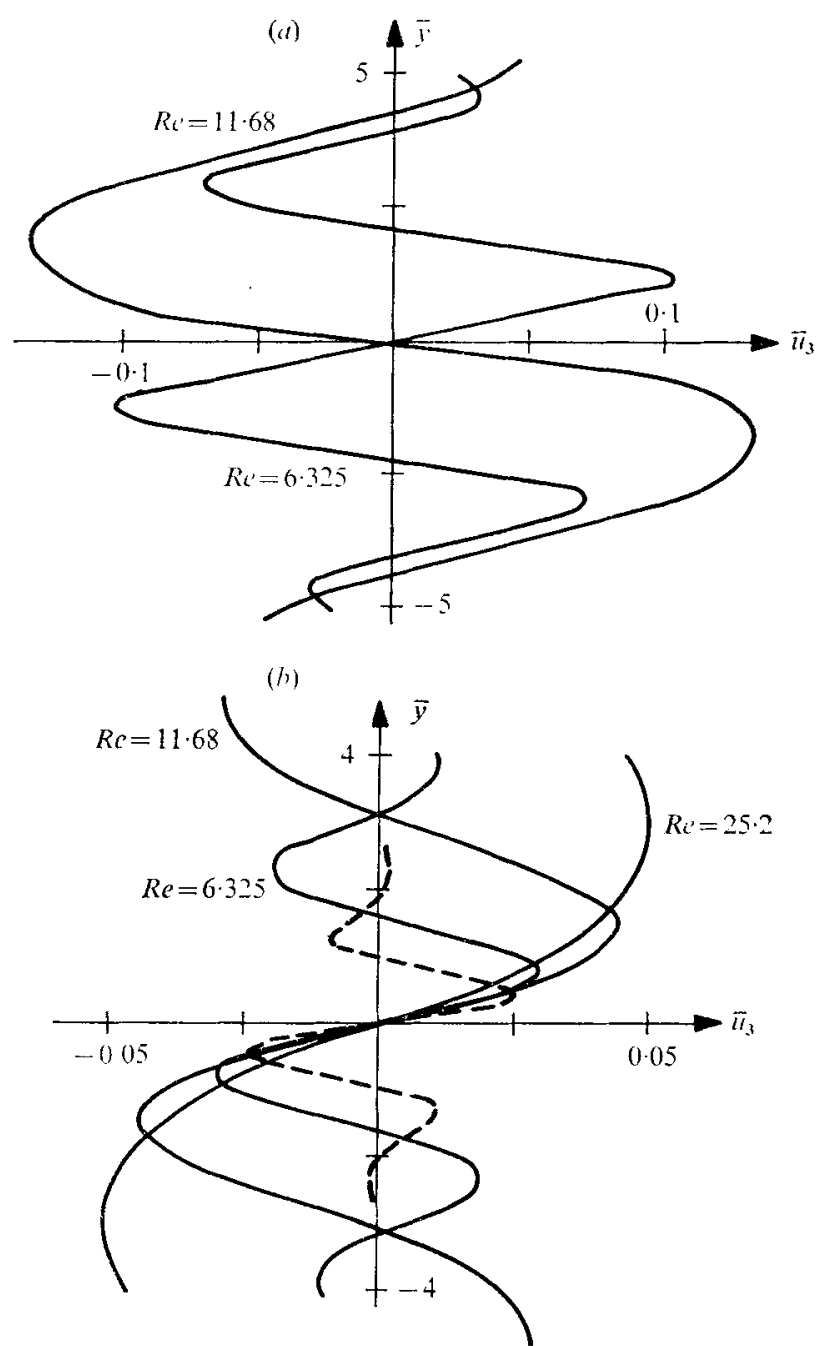

FIGURE 6. $\bar{u}_{3}\left(\equiv 40 R e^{-1} u_{3}\right)$ as a function of $\bar{y}\left(\equiv \frac{1}{40} y R e\right)$ for various Reynolds numbers and $\operatorname{Pr}=0 \cdot 7$. for $R e=6 \cdot 325$.
(a) $\bar{x}=\frac{1}{40}-x R e=2 \cdot 5$.
(b) $\bar{x}=\frac{1}{10} x R e=-2 \cdot 5$.

although it is clear from the far-field similarity profile that, in an averaged sense, the situation is unchanged. As might be expected qualitatively, the far-wake self-similar disturbance flows are strongest for the horizontal momentum and heat sources $\left(O\left(|x|^{-\frac{2}{3}}\right)\right)$ and weakest for the vertical momentum source $\left(O\left(|x|^{-\frac{6}{3}}\right)\right)$ with the heat dipole falling in between.

\section{Oseen solutions}

From equations (10) and (11) for the functions $I_{u i}, i=1,2_{s}, 2_{d}, 3$, we can ascertain the general behaviour of the horizontal velocity components in the Oseen solution regime using the characteristics of the poles $s_{1}, s_{2}, \ldots, s_{6}$, as indicated 


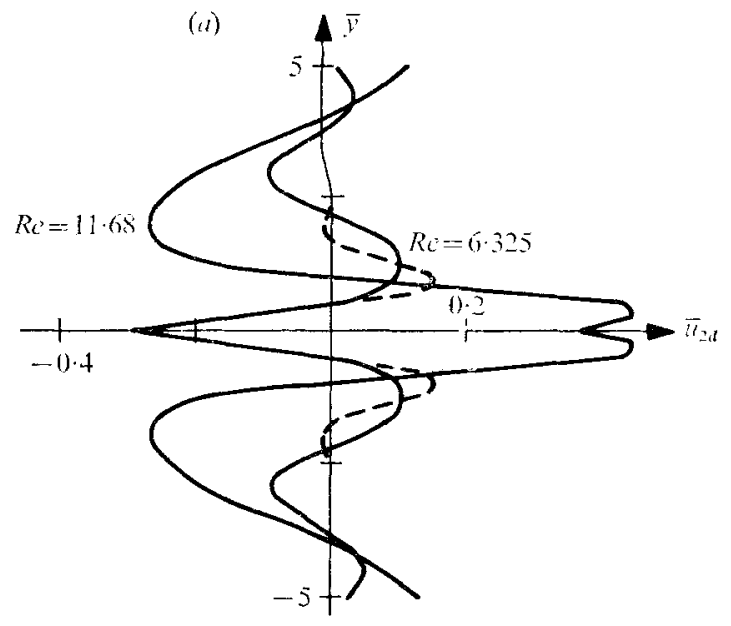

(b)

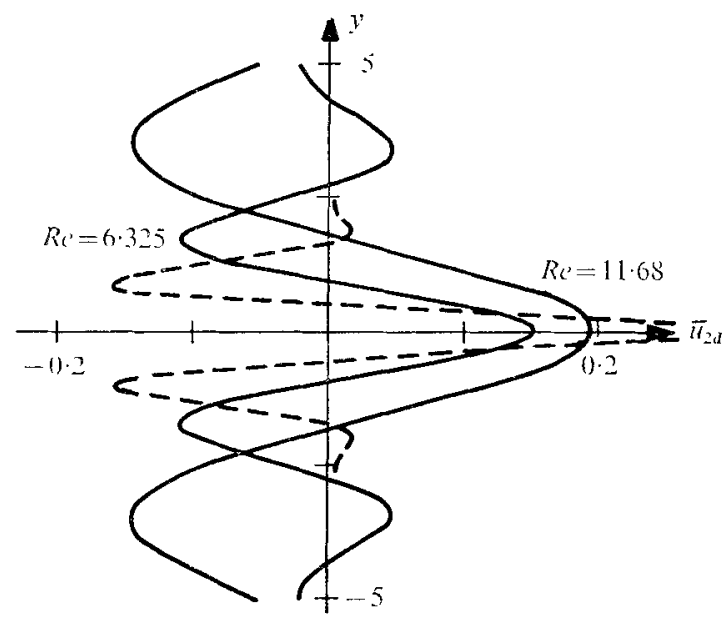

FIGURE 7. $\bar{u}_{2 d}\left(\equiv 40 R e^{-1} u_{2 d}\right)$ as a function of $\bar{y}\left(\equiv \frac{1}{40} y R e\right.$ ) for various Reynolds numbers and $\operatorname{Pr}=0 \cdot 7$. — Oseen solutions for $R e=6 \cdot 325,11 \cdot 68 ;-\ldots-$ self-similar solution for $R e=6 \cdot 325$.
(a) $\bar{x}=\frac{-1}{40} x R e=2 \cdot 5$.
(b) $\bar{x}=-\frac{1}{40} x R e=-2 \cdot 5$.

earlier. The case of a horizontal momentum source was discussed by Janowitz (1967). However, the general features of the horizontal velocity profiles are clearly common to all four of the singularity types which we have considered, since the general solution forms are similar and the behaviour of the poles is independent of the singularity type.

Downstream, in all four cases, $I_{u_{i}}$ displays terms which are both wavelike in $x$ owing to the complex conjugate poles $s_{2}$ and $s_{3}$ for $0 \leqslant k_{2}<k^{*}$, and exponential owing to the real poles $s_{1}$ for $k_{2} \geqslant 0$ and $s_{2}$ and $s_{3}$ for $k_{2} \geqslant k_{2}^{*}$. For moderate distances downstream, all terms are significant in general, indicating the presence of lee-waves in the overall velocity field. Since the real and imaginary parts of $s_{2}$ both increase with decreasing $R e,(10 a)$ indicates that increasing the 

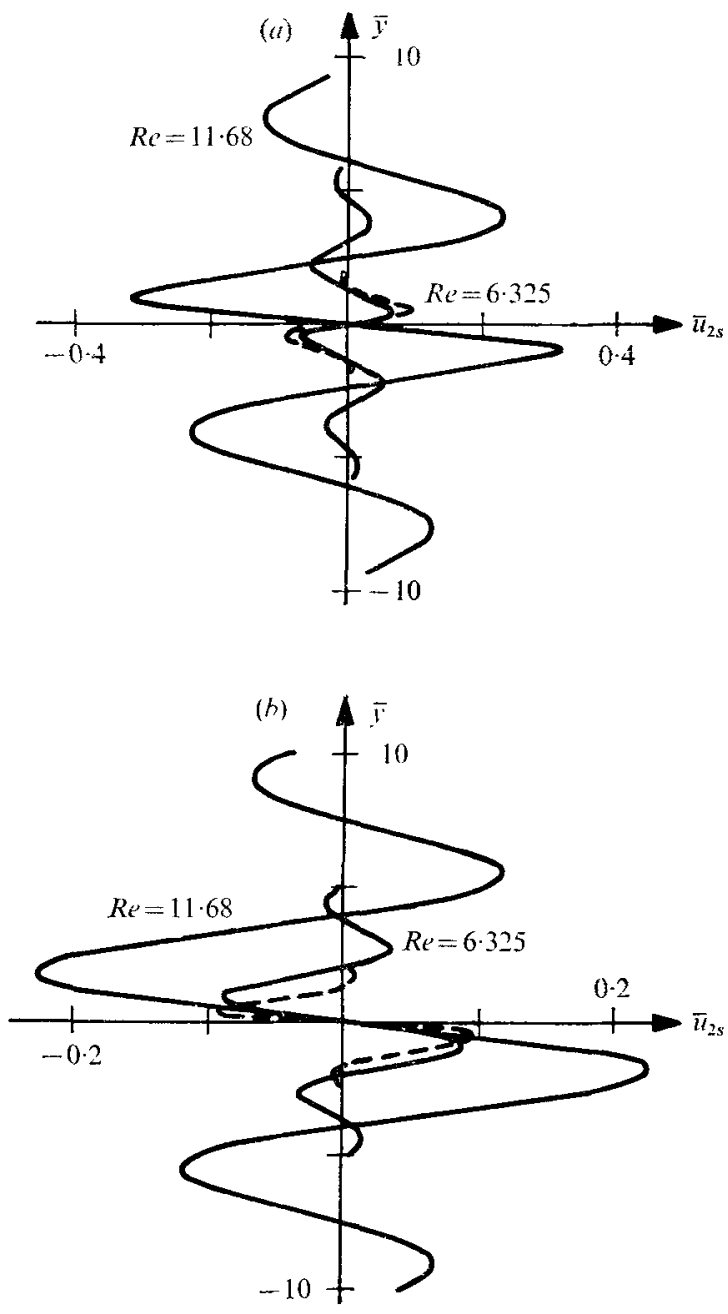

FIGURE $8 \cdot \bar{u}_{2 s}\left(\equiv 40 R e^{-1} u_{2 s}\right)$ as a function of $\bar{y}\left(\equiv \frac{1}{40} y R e\right)$ for various Reynolds numbers and $\operatorname{Pr}=0 \cdot 7$. - Oseen solutions for $R e=6 \cdot 325,11 \cdot 68 ;-\ldots$, self-similar solution for $R e=6 \cdot 325$.

degree of ambient stratification will cause a decrease in the amplitude and wavelength of these lee-waves. Both the pure exponential and wavelike terms decrease in magnitude with increasing downstream distances. However, since $s_{1}<s_{2 R}$, very far downstream the first term of $(10 a)$ dominates $I_{u_{i}}$ yielding the diffusive self-similar wake solutions which were discussed in the preceding section.

Upstream, $I_{u_{i}}$ is dominated for each singularity type by the term containing the real root $s_{4}$. Thus, upstream the horizontal velocity distributions do not exhibit any appreciable wavelike character with respect to $x$. However, as we shall see from the evaluation of the full solutions, they do exhibit an alternating jet structure with $y$ which merges very far upstream with the diffusive selfsimilar wake solutions. 
Finally, when the fluid is homogeneous, a similar analysis yields all real poles for $k_{2} \geqslant 0$, thus indicating a complete absence of wavelike terms in the horizontal velocity components.

In spite of these general features, which are common to all four singularity types, substantial detailed differences do exist in the Oseen region owing to the contrasts in the flows which are induced nearer the body. Of course, we have seen differences even further out in the self-similar regime which we considered in the last section, and it is inconceivable that this intermediate region would not at least yield differences of comparable severity. The horizontal velocity component profiles, which we shall examine first, were calculated by integrating (6) for $\zeta=u_{i}, i=1,2_{s}, 2_{d}, 3$.

We begin by considering the variations in the upstream and downstream profiles with varying horizontal distance from the body (i.e. the origin), and a constant degree of ambient stratification. Profiles for the four singularity types are shown in figures 1-4, together with the self-similar profiles which we discussed earlier. For each singularity type, the Oseen and self-similar profiles both exhibit the characteristic alternating jet structure which has been previously noted for $u_{1}$ by Long (1962) in the self-similar regime, and by Janowitz (1967) for the Oseen region. These profiles broaden vertically and weaken for increasing distances from the disturbances because of the influence of viscosity. Furthermore, as expected qualitatively, the deviations of the full Oseen solutions from the corresponding self-similar profiles decrease with increasing distances from the singularities. Comparison of the Oseen and self-similar profiles for a specific value of $|x|$ shows that the former are more spread out vertically and have magnitudes which are smaller upstream and larger downstream than would be the case if the flow development were everywhere self-similar (i.e. with negligible inertia). Comparing the Oseen profiles for the four singularity types shows differences in the magnitudes and symmetry about $y=0$ which are similar to those exhibited by the similarity solutions. Thus, disturbance flows symmetric about $y=0$ are created by both the horizontal momentum source and the vertically oriented heat dipole. Conversely, antisymmetric disturbance flows are created by both the vertical momentum source, and the heat source as the vertically induced motion near the body is converted by the ambient density stratification into horizontal motions. Upstream the profiles are qualitatively similar at all stations (though spread out and weakened as described before). However, downstream one striking feature of the horizontal velocity profiles is the apparent fact that they may be completely reversed in sign from one streamwise position to the next.

The effect of varying the degree of ambient stratification on the horizontal velocity component for fixed streamwise position $(\bar{x}=x \operatorname{Re} / 40= \pm 2 \cdot 5)$ is shown in figures 5-8. As suggested by the qualitative arguments at the beginning of this section, the velocity profiles for all cases, both upstream and downstream, are decreased in magnitude and strongly compressed vertically, as the degree of stratification is increased. In contrast to the self-similar profiles, which are qualitatively similar for all $R e$, the Oseen profiles downstream can again vary strongly with $R e$, sometimes showing a complete reversal of sign. Finally, by 
comparing figures 1-4 for $R e=11 \cdot 68$ and figures $5-8$ for $R e=6 \cdot 325$, it can be seen that the deviations of the Oseen solutions from their self-similar forms, at a fixed streamwise position, are decreased with decreasing $R e$. This feature is expected qualitatively and simply reflects the decreased importance of fluid inertia for lower values of $R e$.

To date, nearly all of the theoretical work on the motion of bodies through a stratified fluid has concentrated on the horizontal velocity component (ef. Long 1962; Janowitz 1967, 1968). In order to gain additional physical insight into the alternating jet structure and other interesting features of the horizontal velocity profiles, we also carried out explicit calculations of the disturbance stream-function fields for the four types of singularities. Typical results are illustrated in figures 9-12 for various degrees of ambient stratification. The length scale (both horizontal and vertical) in each case is indicated by marking the points $\bar{x}=0$ and $\bar{x}=3$ for $\bar{y}=0$ in part $(a)$ of the figure, with the origin being denoted on all plots as a large dot. The disturbance flow consists of a pattern of rotors of finite vertical thicknesses for stratified fluids $\left(\gamma^{\prime}>0\right)$ and an open streamline pattern for homogeneous fluids $\left(\gamma^{\prime}=0\right)$. As explained by List (1971) for creeping flows, the rotor structure for stratified fluids is a simple consequence of the fact that the induced flows (and associated return flows) are constrained by the ambient stratification to regions of finite vertical extent.

Both upstream and downstream of each singularity type, the alternating jet structure of the horizontal velocity profiles for $\gamma^{\prime}>0$ (and its absence for $\gamma^{\prime}=0$ ) may now be associated with the existence for $\gamma^{\prime}>0$ (and absence for $\gamma^{\prime}=0$ ) of closed-streamline rotor flows, with each rotor being of finite vertical extent and rotating in the opposite direction from its nearest neighbours. For each singularity type, the rotors increase in number and decrease in strength and size for increasing amounts of stratification; this is reflected in the decreasing magnitude and vertical wavelength of the alternating jet profiles for the horizontal velocity components. Finally, the disturbance flow downstream can also exhibit a series of alternating, closed-streamline flow patterns with increasing $x$, whereas upstream the alterations in the sense of rotation are confined to the vertical direction. Hence, the occurrence in some cases of changes in shape (e.g. the reversal in sign of $u_{3}, u_{2 d}$, and $u_{2 s}$ ) of the alternating jet structure with increasing downstream distances (or increasing $\gamma^{\prime}>0$ ) and the contrasting similarity in all of the upstream profile shapes may again be ascribed to the existence of rotors of horizontally finite and semi-infinite extent, respectively, in the disturbance flows.

Our Oseen solutions for the horizontal and vertical momentum sources can be compared with the Stokes-flow calculations of List (1971) for $\gamma^{\prime}>0$. List found the rotors to be symmetric (or antisymmetric) upstream and downstream. In contrast, the inclusion of the Oseen-type convection term results in a skewing of the rotors towards the downstream direction, as observed experimentally by Long (1959). Our solutions also show the existence of a whole family of rotors, thus allowing reversals in the flow direction as one moves in either the vertical or horizontal (downstream) direction. Thus, we obtain jets for the horizontal velocity profiles whose number depends on $R e$ and $x$, as found in Pao's experiments (1968), and which may change drastically in shape between different 
(a)

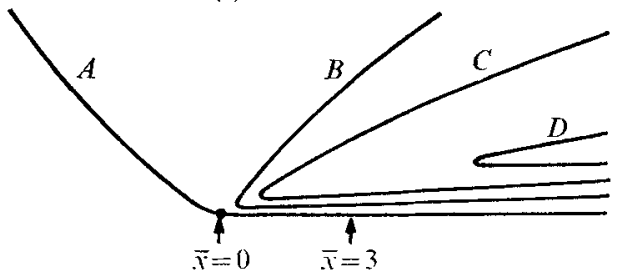

(h)
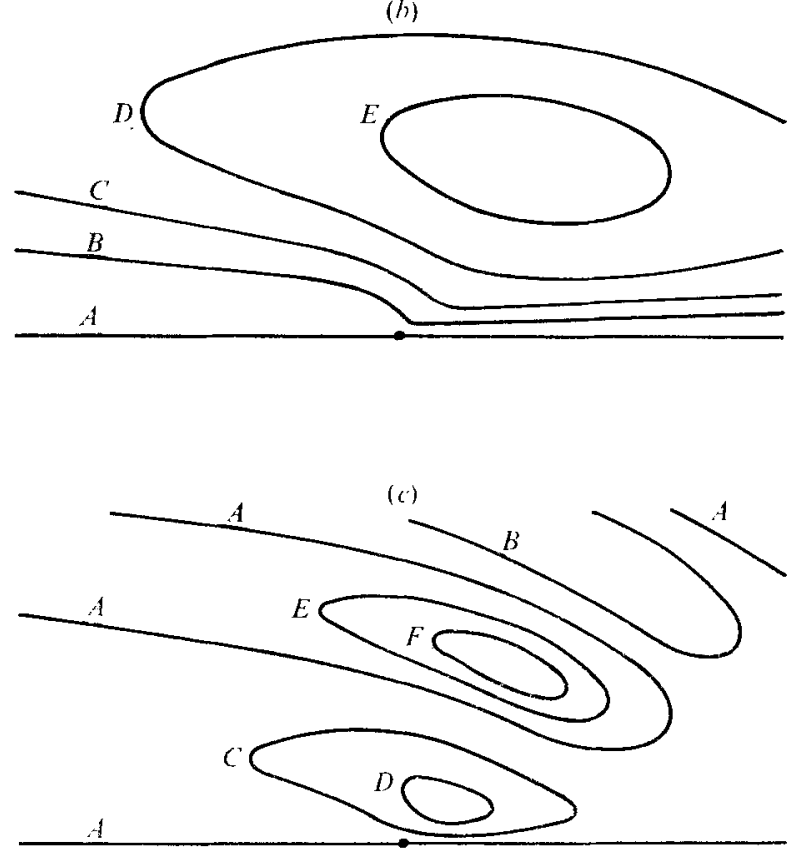

(d)

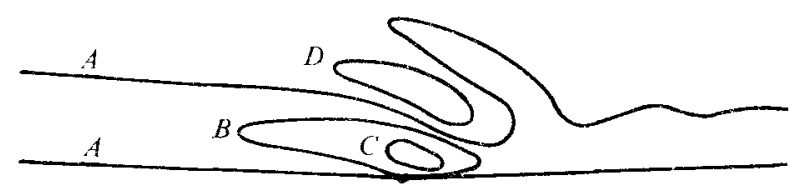

Figure 9. Streamlines for a horizontal momentum source with $\operatorname{Pr}=0 \cdot 7$. (a) $\gamma^{\prime}=0$; $\bar{\psi}_{1}(\bar{x}, \bar{y})=\psi_{1}\left(\frac{1}{40} x_{\phi}, \frac{1}{40} y_{\phi}\right): A=-0 \cdot 10, B=-0 \cdot 35, C=-0.40, D=-0.45$. (b) $R e=$ $25 \cdot 2 ; \psi_{1}\left(\bar{x}_{,}, \bar{y}\right)=\psi_{1}(x / 1.586, y / 1.586): A=-0.001, B=-0.4, C=-0.6, D=-0.8$, $E=-1.0 . \quad(c) \quad R e=11 \cdot 68 ; \bar{\psi}_{1}(\bar{x}, \bar{y})=\psi_{1} \quad(x=3.418 \bar{x}, y=3.418 \bar{y}): A=-0.001$, $B=-0 \cdot 1, C=-0 \cdot 3, D=-0 \cdot 5, E=0 \cdot 10, F=0 \cdot 15$. (d) $\operatorname{Re}=6 \cdot 325 ; \bar{\psi}_{1}(\bar{x}, \bar{y})=$ $\psi_{1}(x=6 \cdot 325 \bar{x}, y=6 \cdot 325 \bar{y}): A=-0 \cdot 05, B=-0 \cdot 15 ; C=-0 \cdot 30, D=0 \cdot 4$. 

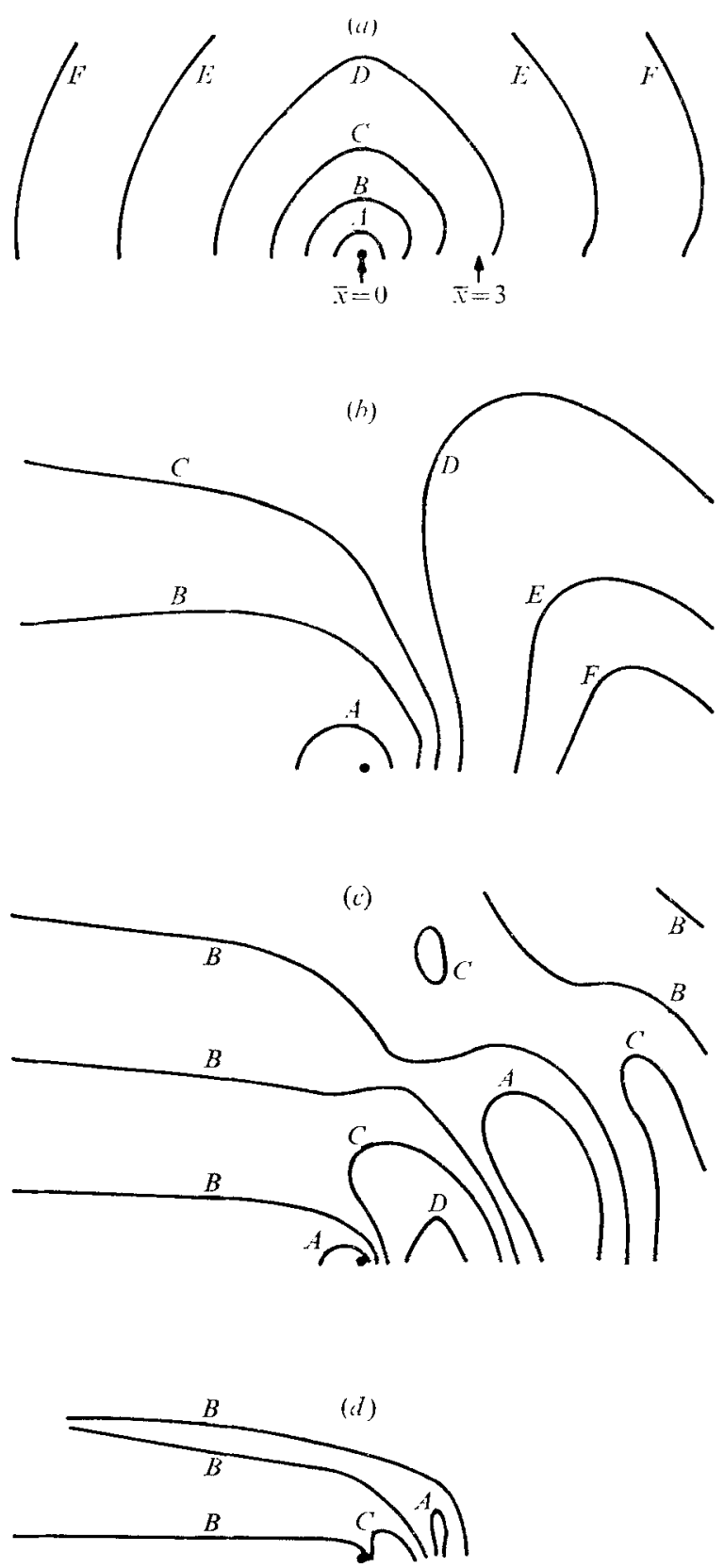

Figure 10. Streamlines for a vertical momentum source with $\operatorname{Pr}=0 \cdot 7 .(a) \gamma^{\prime}=0$; $\overline{\psi_{3}}(\bar{x}, \bar{y})=\psi_{3}\left(x_{\phi}=40 \bar{x}, y_{\phi}=40 \bar{y}\right): A=-0 \cdot 466, B=-0 \cdot 316, C=-0 \cdot 216, D=-0 \cdot 116$, $E=-0 \cdot 016, F=0.054 .(b) R e=25 \cdot 2 ; \bar{\psi}_{3}(\bar{x}, \bar{y})=\psi_{3}(x=1.586 \bar{x}, y=1.586 \bar{y}): A=-0.30$, $B=-0 \cdot 10, C=-0.001, D=0 \cdot 10, E=0 \cdot 30, F=0 \cdot 40 .(c) R e=11 \cdot 68 ; \bar{\psi}_{3}(\bar{x}, \bar{y})=$ $\psi_{3}(x=3 \cdot 418 \bar{x}, y=3 \cdot 418 \bar{y}): A=-0 \cdot 10, B=-0 \cdot 001, C=0 \cdot 10, D=0 \cdot 30 .(d) R e=6 \cdot 325$; $\bar{\psi}_{3}(\bar{x}, \bar{y})=\psi_{3}(x=6 \cdot 325 \bar{x}, y=6 \cdot 325 \bar{y}): A=-0 \cdot 10, B=-0 \cdot 001, C=0 \cdot 125$. 

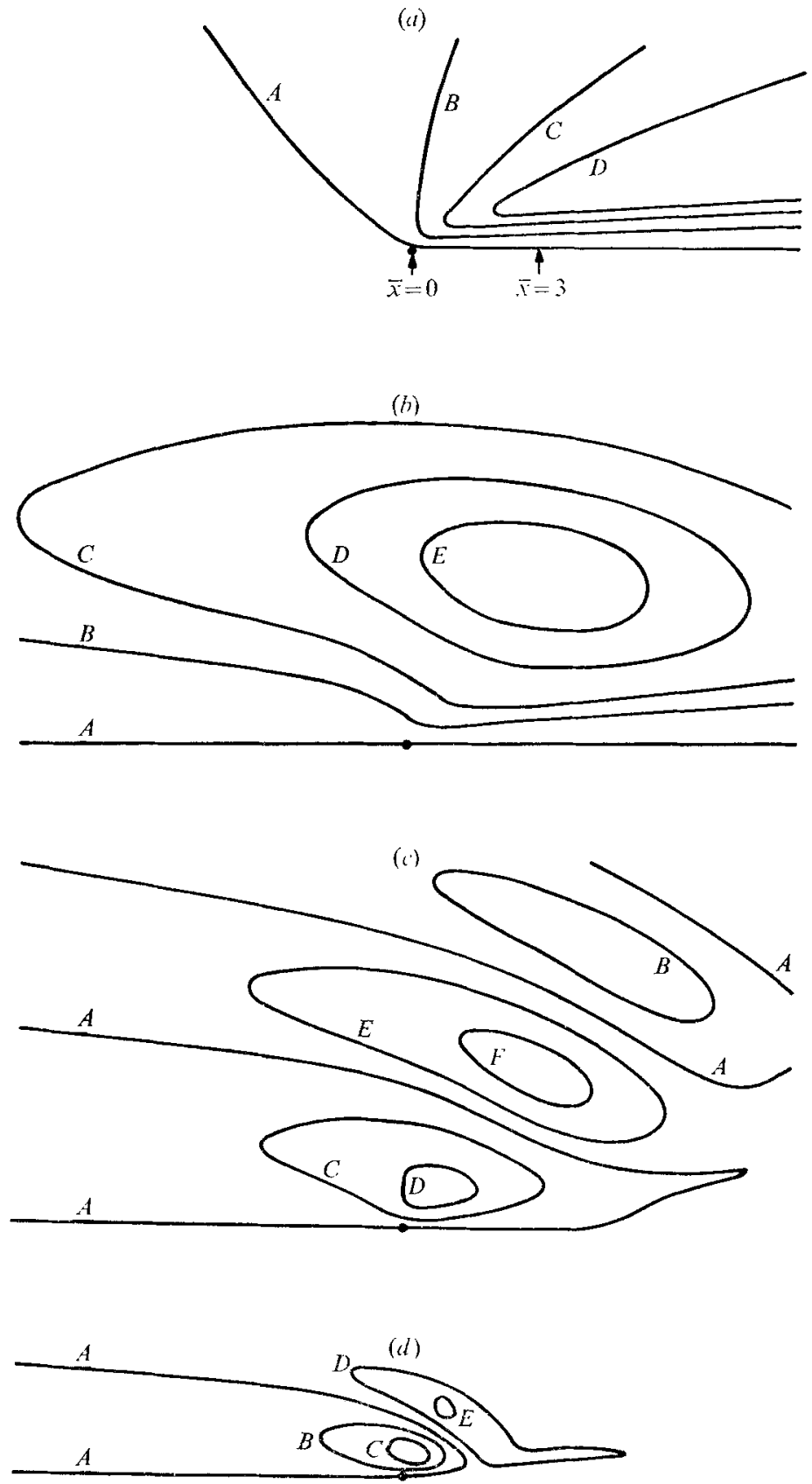

FigURe 11. Streamlines for a vertically oriented heat dipole with $\operatorname{Pr}=0.7$. (a) $\gamma^{\prime}=0$; $\bar{\psi}_{2 d}(\bar{x}, \bar{y})=\psi_{2 a}\left(x_{\phi}=40 \bar{x}, y_{\phi}=40 \bar{y}\right): A=0 \cdot 10, B=0.25, C=0 \cdot 35, D=0.40 . \quad(b)$ $R e=25.2 ; \bar{\psi}_{2 d}(\bar{x}, \bar{y})=\psi_{2 d}(x=1.586 \bar{x}, y=1.586 \bar{y}): A=0.001, B=0.4, C=0.6$, $D=0 \cdot 8, E=0.9$. (c) $\operatorname{Re}=11 \cdot 68 ; \bar{\psi}_{2 d}(\bar{x}, \bar{y})=\psi_{2 d}(x=3 \cdot 418 \bar{x}, y=3.418 \bar{y}): A=0.001$, $B=0.1, C=0.2, D=0.4, E=-0.10, F=-0.20 . \quad(d) R e=6.325 ; \bar{\psi}_{2 a}(\bar{x}, \bar{y})=$ $\psi_{2 a}(x=6.325 \bar{x}, y=6.325 \bar{y}): A=0.001, B=0.10, C=0.20, D=-0.05, E=-0.10$. 


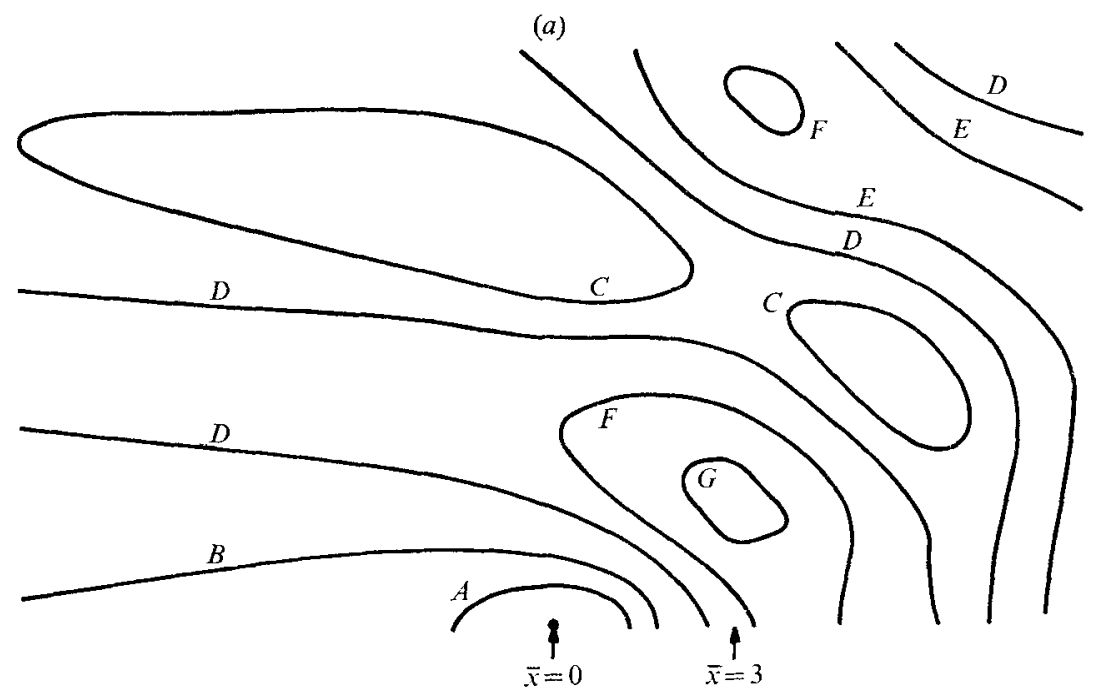

(b)

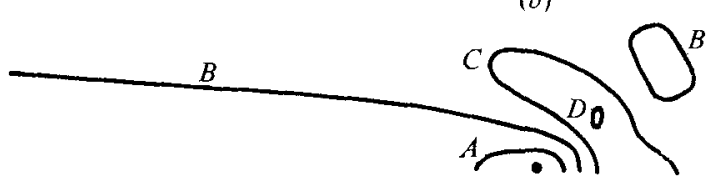

FIGURE 12. Streamlines for a heat source with $\operatorname{Pr}=0 \cdot 7$. (a) $\operatorname{Re}=11 \cdot 68 ; \bar{\psi}_{2 s}(\bar{x}, \bar{y})=$ $\psi_{2 s}(x=3.418 \bar{x}, y=3 \cdot 418 \bar{y}) / 3 \cdot 418: A=0.5, B=0 \cdot 3, C=0 \cdot 1, D=0.001, E=-0 \cdot 1$, $F=-0.2, G=-0.4$. (b) $R e=6 \cdot 325 ; \bar{\psi}_{2 s}(\bar{x}, \bar{y})=\psi_{2 s}(x=6 \cdot 325 \bar{x}, y=6 \cdot 325 \bar{y}) / 6 \cdot 325:$ $A=0.10, B=0.02, C=-0.05, D=-0.10$.

downstream positions or different Reynolds numbers. On the other hand, for $y>0$ and $\gamma^{\prime}>0$, List found only one rotor for $\psi_{1}$ (corresponding to only two jets for $u_{1}$ ) and a system of three rotors for $\psi_{3}$ which change rotation directions vertically, but not horizontally. Thus the creeping-flow solutions could not account for the changes in shape of the downstream alternating jet structure or for other differences between the upstream and downstream profiles.

Finally, the total streamline plots, created by adding the disturbance flow streamlines associated with a particular singularity to the ambient uniform flow streamlines $\tilde{\psi}=y$, have been obtained by Robertson (1975) and confirm the qualitative interpretation of the presence of a periodic structure in $u_{i}, i=1,2_{s}$, $2_{d}, 3$, with respect to $x$ for $\gamma^{\prime}>0$ as indicative of lee-waves. The presence for $\gamma^{\prime}>0$ (and absence for $\gamma^{\prime}=0$ ) of the wavelike structure downstream is associated with the corresponding presence for $\gamma^{\prime}>0$ (and absence for $\gamma^{\prime}=0$ ) of a series of alternating rotors with increasing downstream distances in the disturbance stream-function fields. 


\section{Appendix. Distance requirements for valid solutions}

Having discussed in detail both the self-similar and Oseen solutions, we should also like to estimate $a$ priori the distances from each type of singularity required for the two solutions to be valid. For the Oseen solution regime, we have considered distances so large that the Oseen linearization of the convective operator is applicable; i.e. $|u| \ll 1$ or

$$
|u| \leqslant 0 \cdot 1
$$

where $\leqslant 0 \cdot 1$ corresponds, for numerical purposes, to $\ll 1$; qualitatively, similar results would be obtained if a number other than 0.1 were chosen. Since we do not have closed-form Oseen solutions for the horizontal velocity components, as they have to be evaluated by a numerical integration, we cannot calculate a priori analytic expressions for the distances required from the relation (A 1). However, explicit expressions which provide a conservative estimate of these distances can be obtained by substituting the closed-form self-similar expressions into the Oseen condition (A 1), thereby yielding the distance requirements:

$$
\begin{aligned}
& |x| \geqslant 0.61 \operatorname{Re}^{\frac{5}{4}} \operatorname{Pr}^{-\frac{1}{4}} \quad \text { for a horizontal momentum source } \\
& |x| \geqslant 0.53 \operatorname{Re}^{\frac{1}{2}} \operatorname{Pr}^{-\frac{1}{4}} \quad \text { for a vertical momentum source, }
\end{aligned}
$$

and $|x| \geqslant 0.50 R e^{\frac{1}{2}} P r^{\frac{1}{2}} \quad$ for a heat source or vertically oriented heat dipole, when $K_{1}=K_{2}=K_{3}=1$. Figure 13 illustrates the estimates of the regimes of validity for the Oseen solutions due to each singularity type for $\mathrm{Pr}=0.7$. For the horizontal momentum source, these estimates are seen to approximate well the actual regimes of validity obtained $a$ posterior $i$ by calculating the Oseen solution at various values of $|x|$ to find the value of $|x|$ which must be exceeded for the Oseen condition (A 1) to apply.

The behaviour of the similarity solution regime is governed by (15); hence, in addition to the relation (A 1), additional distance restrictions on the self-similar solutions are imposed by the conditions $|\partial u / \partial x| \ll|\partial p / \partial x|,\left|\partial^{2} u / \partial x^{2}\right| \ll\left|\partial^{2} u / \partial y^{2}\right|$, $|\partial v / \partial x| \ll|\partial p / \partial y|,\left|R e^{-1} \partial^{2} v / \partial y^{2}\right| \ll|\partial p / \partial y|,\left|R e^{-1} \partial^{2} v / \partial x^{2}\right| \ll|\partial p / \partial y|,|\partial \vartheta / \partial x| \ll|v|$, and $\left|\partial^{2} \vartheta / \partial x^{2}\right| \ll\left|\partial^{2} \vartheta / \partial y^{2}\right|$. The most restrictive distance requirement determined from all the above conditions is $\dagger$

$$
|x| \gg \operatorname{Re}^{2} \operatorname{Pr}^{-1}
$$

for each singularity type and $\operatorname{Pr}=0 \cdot 7$, resulting in each case from the condition $|\partial u / \partial x| \ll|\partial p / \partial x|$. For the self-similar solutions, the regime of validity, which is further from each singularity than the corresponding Oseen solution regime as expected, is illustrated in figure 13. As expected, for decreasing $R e$, the region in which the Oseen solution is valid but the self-similar solution is not decreases in size.

It is also of interest to estimate the actual distances required for the selfsimilar and Oseen solutions to be valid corresponding to experimental conditions reported previously. For the experiment in figure 1 of Laws \& Stevenson (1972),

$\dagger$ In the large stratification (low Re) limit, Freund \& Meyer (1972) also derived this distance requirement for the validity of the similarity solution in which the detailed body geometry is unimportant. 


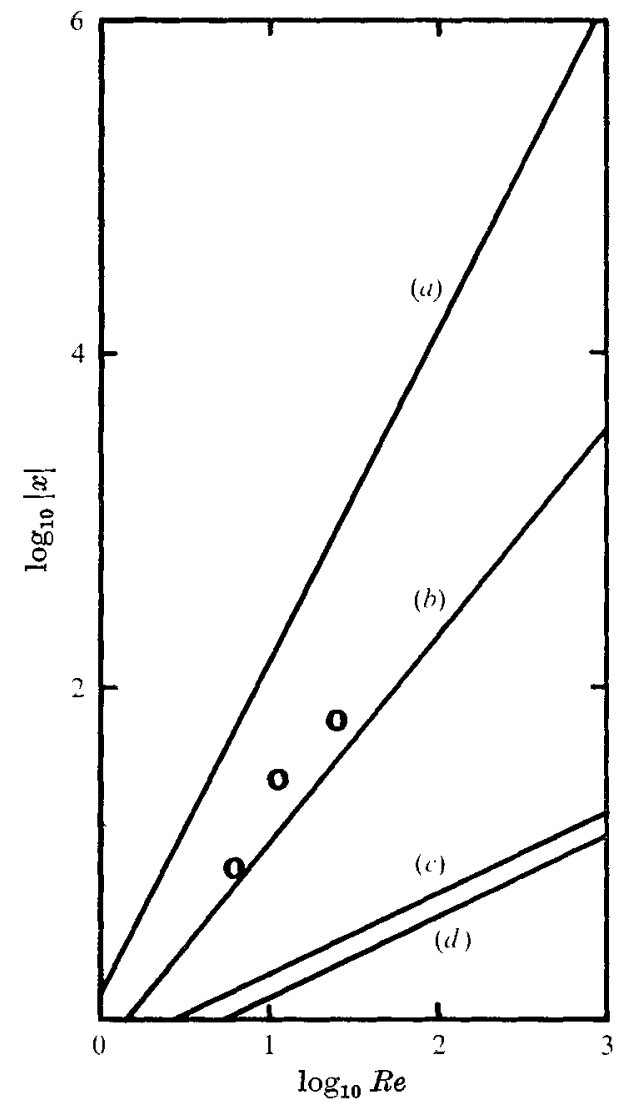

FIgURE 13. Minimum distance requirements for valid solutions $; \operatorname{Pr}=0 \cdot 7 .(a)|x|=\operatorname{Re}^{2} \operatorname{Pr}^{-1}$;

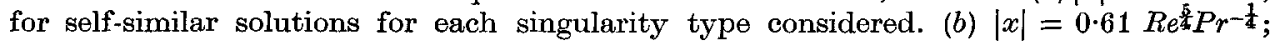
estimate for Oseen solution for horizontal momentum source with $K_{1}=1$. (c) $|x|=$ $0.53 \operatorname{Re}^{\frac{1}{2}} \mathrm{Pr}^{-\frac{1}{4}}$; estimate for Oseen solution for vertical momentum source with $K_{3}=1$. (d) $|x|=0.50 \operatorname{Re}^{\frac{1}{2}} \operatorname{Pr}^{\frac{1}{2}}$; estimate for Oseen solution for heat source or vertically oriented heat dipole singularity with $K_{\mathrm{z}}=1$. The points $O$ represent the minimum distance requirements calculated numerically from the Oseen solution for a horizontal momentum source with $K_{1}=1$.

$\nu_{\infty}=\mu_{\infty} / \rho_{\infty}=1.15 \mathrm{~mm}^{2} \mathrm{~s}^{-1}, u_{\infty}=0.032 \mathrm{~mm} \mathrm{~s}^{-1}$, and $\left(\beta T_{\infty} \gamma^{\prime}\right)^{-1}=5.5 \mathrm{~m}$. From figure 1 of the present work we see that the profiles are identical at $x=854 \cdot 5$, which corresponds to a distance of $255 \mathrm{~mm}$ in the system of Laws \& Stevenson. The diameter of the cylinder used in that study was $25.4 \mathrm{~mm}$, so that selfsimilarity appears to be achieved about 10 diameters downstream.

\section{REFERENCES}

BrowaNd, F. K. \& WINANT, C. D. 1972 Blocking ahead of a cylinder moving in a stratified fluid: an experiment. Geophys. Fluid Dyn. 4, 29.

Carsuaw, H. S. \& Jaeger, J. C. 1959 Conduction of Heat in Solids, p. 271. Oxford University Press.

Frednd, D. D. \& Meyer, R. E. 1972 On the mechanism of blocking in a stratified fluid. J. Fluid Mech. 54, 719 . 
Graebes, W. P. 1969 On the slow motion of bodies in stratified and rotating fluids. Quart.J. Mech. Appl. Math. 22, 39.

JANowitz, G. S. 1967 On wakes in stratified fluids. Dept. Mech., The Johns Hopkins University, Tech. Rep. no. 22 (ONR Series), Nonr-4010(01).

JANowitz, G. S. 1968 On wakes in stratified fluids. J. Fluid Mech. 33, 417.

JANowitz, G.S. 1971 The slow transverse motion of a flat plate through a non-diffusive stratified fluid. J. Fluid Mech. 47, 171.

Laws, P. \& Stevenson, T. N. 1972 Measurements of a laminar wake in a confined stratified fluid. J. Fluid Mech. 54, 745.

List, E. J. 1971 Laminar momentum jets in a stratified fluid. J. Fluid Mech. 45, 561.

LoNG, R. R. 1959 The motions of fluids with density stratification. J. Geophys. Res. 64, 2151.

LoNg, R. R. 1962 Velocity concentrations in stratified fluids. J. Hydraul. Div. A.S.C.E. $88,9$.

Moone, D. W. \& Safrmar, P. G. 1969 The structure of free vertical shear layers in a rotating fluid and the motion produced by a slowly rising body. Phil. Trans. A 264, 597.

Pao, Y.-H. 1968 Laminar flow of a stably stratified fluid past a flat plate. J. Fluid Mech. $34,795$.

Roвertson, G. E. 1975 Ph.D. dissertation, California Institute of Technology.

Robertson, G. E., Seinfeld, J.H. \& Leal, L. G. 1973 Combined forced and free convection flow past a horizontal flat plate. A.I.Ch.E. J. 19, 998. 